\title{
Photoelastic modulator non-idealities in magneto-optical polarization measurements
}

\author{
Stefaan Vandendriessche and Thierry Verbiest \\ Molecular Imaging and Photonics, KU Leuven, Celestijnenlaan 200 D bus 2425, B-3001 Heverlee, Belgium
}

\begin{abstract}
Modifying and detecting the polarization of light is increasingly important in many contexts such as Faraday isolators and electro-optical modulators. In order to control the polarization of light, it is necessary to know the polarization characteristics of the materials used in the applications. To be able to (magneto-)optically characterize novel materials, we designed a setup using a single photoelastic modulator (PEM) to simultaneously detect natural and magnetic circular dichroism and circular birefringence over a large spectral range. We then theoretically analyzed and experimentally characterized the effect of non-idealities in the PEM on the setup and the resulting data. Our results demonstrate an influence of PEM non-idealities on the measured signals, resulting in non-negligible mixing of circular birefringence and circular dichroism signals. Our measurements of the wavelength dependence of these non-idealities reveal larger non-idealities towards shorter wavelengths. These results illustrate the necessity to take PEM non-idealities into account when working with PEMs, especially at shorter wavelengths or when dealing with signals spanning different orders of magnitude. PEM non-idealities, while frequently neglected in experimental setup design and theoretical derivations, are expected to be more complicated and possibly exert a larger influence on obtained results for experimental setups with multiple PEMs.
\end{abstract}

Keywords: photoelastic modulator, PEM, Faraday rotation,circular birefringence,circular dichroism,magnetic circular dichroism, bessel function,polarization

\section{INTRODUCTION}

Polarization is an important fundamental property of electromagnetic waves. ${ }^{1}$ Detecting and manipulating the polarization of light is essential for both fundamental research as well as for applications. ${ }^{2-9}$ A variety of optical elements exist to manipulate the polarization of UV to infrared light. ${ }^{10-12}$ It can be useful to be able to modulate this polarization, and photoelastic modulators modulate the polarization of light at high frequencies, based on the stress-dependence of birefringence in certain materials. ${ }^{13}$ Photoelastic modulators have been used for well over 40 years, ${ }^{14}$ finding applications in a wide variety of fields such as protein adsorption, ${ }^{15}$ high-speed semiconductor growth monitoring, ${ }^{16}$ liquid crystal characterization, ${ }^{17}$ biosensing, ${ }^{18}$ semiconductor analysis, ${ }^{19,20}$ following catalysis ${ }^{21}$ and magneto-optical characterization of (nano)materials. ${ }^{22-24}$

When a magnetic field is applied in the direction of propagation of light, the complex refractive indices for left- and right-circularly polarized light are no longer equal. ${ }^{25-28}$ The difference in the real part of the refractive indices results in Faraday rotation. Faraday rotation can be observed as the rotation of the polarization of linearly polarized light in a magnetic field along the direction of propagation of light. It is described by

$$
\theta=V B L
$$

where $\theta$ is the rotation of the polarization of light, $V$ is the Verdet constant, a wavelength-dependent material parameter, $B$ is the magnetic field in the direction of light and $L$ is the distance light travels through the material in the magnetic field. ${ }^{29}$ The difference in the imaginary part of the refractive indices for left- and right-circularly polarized light manifests itself as magnetic circular dichroism (MCD) $)^{30-32}$

$$
\Delta A=\frac{A_{L C P}-A_{R C P}}{A_{L C P}+A_{R C P}}
$$

Send correspondence to stefaan.vandendriessche@fys.kuleuven.be

Polarization Science and Remote Sensing VI, edited by Joseph A. Shaw, Daniel A. LeMaster, Proc. of SPIE Vol. 8873, 88730Z · (C) 2013 SPIE · CCC code: 0277-786X/13/\$18 - doi: 10.1117/12.2022211 
where $\triangle A$ is the MCD signal and $A_{L C P}$ and $A_{R C P}$ are respectively the absorption of left- and right-hand circularly polarized light.

We designed a setup using a single photoelastic modulator (PEM) to simultaneously detect circular dichroism, both natural and magnetic, circular birefringence and Faraday rotation over a large spectral range. These optical effects have many uses in fundamental research ${ }^{33}$ and applications, ${ }^{34}$ both for chiral ${ }^{35,36}$ and achiral ${ }^{37-40}$ systems. For the analysis of simple optical systems it is not always necessary to even take into account the polarization of light. ${ }^{41-43}$ However, polarization dependent samples and experimental setups such as ours require more complex analysis, ${ }^{18,23,44,45}$ and this is done using Stokes vectors and Mueller matrices. ${ }^{46-49}$ Light, both polarized and depolarized, can be described by a Stokes vector

$$
\vec{S}=\left(\begin{array}{c}
S_{0} \\
S_{1} \\
S_{2} \\
S_{3}
\end{array}\right)
$$

A detailed analysis of the Stokes parameters can be found elsewhere. ${ }^{50}$ For our analysis, it is important that $S_{0}=I$ where $I$ is the intensity of the light beam. When light passes through an optical element with Mueller matrix $M_{a}$ and then an optical element with Mueller matrix $M_{b}$, the outgoing light can be described as

$$
\vec{S}_{\text {out }}=M_{b} M_{a} \vec{S}_{\text {in }}
$$

Using Mueller calculus, we theoretically analyze and experimentally characterize the effect of PEM nonidealities on the data measured in our setup. We demonstrate an influence of PEM non-idealities on the measured signals, resulting in non-negligible mixing of circular birefringence and circular dichroism signals. These results illustrate the necessity to take PEM non-idealities into account when working with PEMs, especially at shorter wavelengths or when dealing with signals spanning different orders of magnitude. Moreover, these results can be expanded to other experimental setups such as dedicated CD-spectrometers.

\section{EXPERIMENTAL DETAILS}

In the experimental setup (Figure 1), a laser driven light source (Energetiq LDLS EQ-99) is focussed into a collimated beam. A polarizer polarizes the beam after it passes through a monochromator. The polarized beam passes through a photoelastic modulator (Hinds I/FS50) operating at $50 \mathrm{kHz}$, placed with its main axis 45 degrees relative to the polarization of the light. The beam then passes through a sample in a dc magnet, and after passing through an analyzer at 45 degrees with respect to the first polarizer it is detected by a photomultiplier tube. Two lock-in amplifiers (SR830) detect the resulting signal, one detecting at the fundamental frequency of the PEM $(50 \mathrm{kHz})$, the other detecting the second harmonic $(100 \mathrm{kHz})$. At discrete magnetic fields the signal value of each lock-in amplifier is read out by a personal computer. In the subsequent section, the relation between the $50 \mathrm{kHz}$ and $100 \mathrm{kHz}$ signal and the (magnetic) circular dichroism and the optical rotation and Faraday rotation is derived.

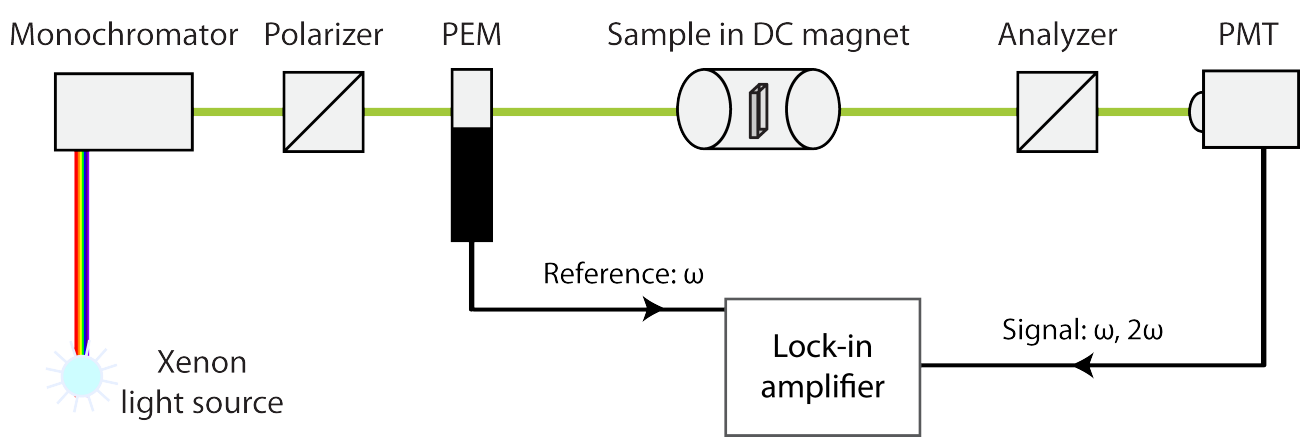

Figure 1. In the experimental setup, a single PEM is used to detect (magnetic) circular dichroism and birefringence. 


\section{THEORETICAL AND EXPERIMENTAL RESULTS}

\subsection{General theoretical derivation}

To be able to describe the setup, knowledge is necessary of the Mueller matrices of the components. For the first polarizer, at 45 degrees, the Mueller matrix is

$$
M_{45 \text { degpolarizer }}=\frac{1}{2}\left(\begin{array}{cccc}
1 & 0 & \pm 1 & 0 \\
0 & 0 & 0 & 0 \\
\pm 1 & 0 & 1 & 0 \\
0 & 0 & 0 & 0
\end{array}\right)
$$

The Mueller matrix for the PEM is

$$
M_{P E M}=\left(\begin{array}{cccc}
1 & 0 & 0 & 0 \\
0 & 1 & 0 & 0 \\
0 & 0 & \cos (\delta) & \sin (\delta) \\
0 & 0 & -\sin (\delta) & \cos (\delta)
\end{array}\right)
$$

where $\delta$ is the dynamic retardation. The Mueller matrix for a general sample is

$$
M_{\text {sample }}=\left(\begin{array}{cccc}
m_{00} & m_{01} & m_{02} & m_{03} \\
m_{10} & m_{11} & m_{12} & m_{13} \\
m_{20} & m_{21} & m_{22} & m_{23} \\
m_{30} & m_{31} & m_{32} & m_{33}
\end{array}\right)
$$

Finally, the Mueller matrix of the horizontal analyzer is

$$
M_{\text {horizontalpolarizer }}=\frac{1}{2}\left(\begin{array}{cccc}
1 & 1 & 0 & 0 \\
1 & 1 & 0 & 0 \\
0 & 0 & 0 & 0 \\
0 & 0 & 0 & 0
\end{array}\right)
$$

For the experimental setup, we can write:

$$
\begin{aligned}
& \vec{S}_{\text {out }}=M_{\text {analyzer }} * M_{\text {sample }} * M_{P E M} * M_{\text {polarizer }} * \vec{S}_{\text {in }} \\
& =\frac{1}{2}\left(\begin{array}{cccc}
1 & 1 & 0 & 0 \\
1 & 1 & 0 & 0 \\
0 & 0 & 0 & 0 \\
0 & 0 & 0 & 0
\end{array}\right)\left(\begin{array}{cccc}
m_{00} & m_{01} & m_{02} & m_{03} \\
m_{10} & m_{11} & m_{12} & m_{13} \\
m_{20} & m_{21} & m_{22} & m_{23} \\
m_{30} & m_{31} & m_{32} & m_{33}
\end{array}\right)\left(\begin{array}{cccc}
1 & 0 & 0 & 0 \\
0 & 1 & 0 & 0 \\
0 & 0 & \cos (\delta) & \sin (\delta) \\
0 & 0 & -\sin (\delta) & \cos (\delta)
\end{array}\right) \frac{1}{2}\left(\begin{array}{cccc}
1 & 0 & 1 & 0 \\
0 & 0 & 0 & 0 \\
1 & 0 & 1 & 0 \\
0 & 0 & 0 & 0
\end{array}\right) \vec{S}_{i n} \\
& =\frac{1}{4}\left(\begin{array}{cccc}
m_{00}+m_{10} & m_{01}+m_{11} & m_{02}+m_{12} & m_{03}+m_{13} \\
m_{00}+m_{10} & m_{01}+m_{11} & m_{02}+m_{12} & m_{03}+m_{13} \\
0 & 0 & 0 & 0 \\
0 & 0 & 0 & 0
\end{array}\right) \\
& *\left(\begin{array}{cccc}
1 & 0 & 0 & 0 \\
0 & 1 & 0 & 0 \\
0 & 0 & \cos (\delta) & \sin (\delta) \\
0 & 0 & -\sin (\delta) & \cos (\delta)
\end{array}\right)\left(\begin{array}{cccc}
1 & 0 & 1 & 0 \\
0 & 0 & 0 & 0 \\
1 & 0 & 1 & 0 \\
0 & 0 & 0 & 0
\end{array}\right) \vec{S}_{i n}
\end{aligned}
$$




$$
\begin{aligned}
& =\frac{1}{4}\left(\begin{array}{cccc}
m_{00}+m_{10} & m_{01}+m_{11} & m_{02}+m_{12} & m_{03}+m_{13} \\
m_{00}+m_{10} & m_{01}+m_{11} & m_{02}+m_{12} & m_{03}+m_{13} \\
0 & 0 & 0 & 0 \\
0 & 0 & 0 & 0
\end{array}\right) *\left(\begin{array}{cccc}
1 & 0 & 1 & 0 \\
0 & 0 & 0 & 0 \\
\cos (\delta) & 0 & \cos (\delta) & 0 \\
-\sin (\delta) & 0 & -\sin (\delta) & 0
\end{array}\right) \vec{S}_{i n} \\
& =\frac{1}{4}\left(\begin{array}{cccc}
a+b \cos (\delta)-c \sin (\delta) & 0 & a+b \cos (\delta)-c \sin (\delta) & 0 \\
a+b \cos (\delta)-c \sin (\delta) & 0 & a+b \cos (\delta)-c \sin (\delta) & 0 \\
0 & 0 & 0 & 0 \\
0 & 0 & 0 & 0
\end{array}\right) \vec{S}_{i n}
\end{aligned}
$$

Where we define

$$
\begin{aligned}
& a=m_{00}+m_{10} \\
& b=m_{02}+m_{12} \\
& c=m_{03}+m_{13}
\end{aligned}
$$

in order to lighten the notation. Because the initial light is unpolarized, $\vec{S}_{i n}=\left(\begin{array}{c}I_{0} \\ 0 \\ 0 \\ 0\end{array}\right)$. This means that

$$
\begin{aligned}
\vec{S}_{\text {out }} & =\frac{1}{4}\left(\begin{array}{cccc}
a+b \cos (\delta)-c \sin (\delta) & 0 & a+b \cos (\delta)-c \sin (\delta) & 0 \\
a+b \cos (\delta)-c \sin (\delta) & 0 & a+b \cos (\delta)-c \sin (\delta) & 0 \\
0 & 0 & 0 & 0 \\
0 & 0 & 0 & 0
\end{array}\right)\left(\begin{array}{c}
I_{0} \\
0 \\
0 \\
0
\end{array}\right) \\
& =\frac{I_{0}}{4}\left(\begin{array}{c}
a+b \cos (\delta)-c \sin (\delta) \\
a+b \cos (\delta)-c \sin (\delta) \\
0 \\
0
\end{array}\right)
\end{aligned}
$$

This means the outgoing light has an intensity detected by the photomultiplier tube

$$
I_{\text {out }}=\frac{I_{0}}{4}(a+b \cos (\delta)-c \sin (\delta))
$$

In order to be able to understand this result, we need to fill in the dynamic retardation of a PEM, which can be written as ${ }^{51}$

$$
\delta(\lambda)=A(\lambda) \sin (\omega t)+\delta_{0}(\lambda)
$$

With $\delta(\lambda)$ the dynamic retardation, $A(\lambda)$ the amplitude of oscillation, $\omega$ the vibration frequency and $\delta_{0}(\lambda)$ the static strain present in the PEM. For an ideal PEM, $\delta_{0}=0$ as there is no static strain. It is however precisely the important non-ideality that $\delta_{0} \neq 0$ that we will focus on later on in the derivation. Applying the trigonometrical sum of angle formulas

$$
\begin{aligned}
& \cos (x+y)=\cos (x) \cos (y)-\sin (x) \sin (y) \\
& \sin (x+y)=\sin (x) \cos (y)+\cos (x) \sin (y)
\end{aligned}
$$

yields

$$
\begin{aligned}
& \cos (\delta)=\cos (A(\lambda) \sin (\omega t)) \cos \left(\delta_{0}(\lambda)\right)-\sin (A(\lambda) \sin (\omega t)) \sin \left(\delta_{0}(\lambda)\right) \\
& \sin (\delta)=\sin (A(\lambda) \sin (\omega t)) \cos \left(\delta_{0}(\lambda)\right)+\cos (A(\lambda) \sin (\omega t)) \sin \left(\delta_{0}(\lambda)\right)
\end{aligned}
$$

Filling this into equation (20) yields 


$$
\begin{aligned}
I & =\frac{I_{0}}{4}\left(a+b\left[\cos (A(\lambda) \sin (\omega t)) \cos \left(\delta_{0}(\lambda)\right)-\sin (A(\lambda) \sin (\omega t)) \sin \left(\delta_{0}(\lambda)\right)\right]\right. \\
& \left.-c\left[\sin (A(\lambda) \sin (\omega t)) \cos \left(\delta_{0}(\lambda)\right)+\cos (A(\lambda) \sin (\omega t)) \sin \left(\delta_{0}(\lambda)\right)\right]\right)
\end{aligned}
$$

This expression can be regrouped to

$$
\begin{aligned}
I & =\frac{I_{0}}{4}\left(a-\left[b \sin \left(\delta_{0}(\lambda)\right)+c \cos \left(\delta_{0}(\lambda)\right)\right] \sin (A(\lambda) \sin (\omega t))\right. \\
& \left.+\left[b \cos \left(\delta_{0}(\lambda)\right)-c \sin \left(\delta_{0}(\lambda)\right)\right] \cos (A(\lambda) \sin (\omega t))\right)
\end{aligned}
$$

In order to understand the signal detected at $\omega$ and $2 \omega$ from equation $(29), \cos (A(\lambda) \sin (\omega t))$ and $\sin (A(\lambda) \sin (\omega t))$ need to be expanded using Bessel functions

$$
\begin{aligned}
& \cos (A \sin (\omega t))=J_{0}(A)+2 \sum_{j=1} J_{2 j}(A) \sin (2 j \omega t) \\
& \sin (A \sin (\omega t))=2 \sum_{j=1} J_{2 j-1}(A) \sin ((2 j-1) \omega t)
\end{aligned}
$$

where $J_{n}$ is the $n$-th Bessel function.

In the experimental setup, the PEM is configured such that $A=2.4048$. This is chosen because $J_{0}(2.4048)=$ 0 , greatly simplifying the derivation and interpretation of experimental results. Using this, and filling equations (30) and (31) into equation (29) yields

$$
\begin{aligned}
I & =\frac{I_{0}}{4}\left(a-\left[b \sin \left(\delta_{0}(\lambda)\right)+c \cos \left(\delta_{0}(\lambda)\right)\right] 2 \sum_{j=1} J_{2 j-1}(A) \sin ((2 j-1) \omega t)\right. \\
& \left.+\left[b \cos \left(\delta_{0}(\lambda)\right)-c \sin \left(\delta_{0}(\lambda)\right)\right] 2 \sum_{j=1} J_{2 j}(A) \sin (2 j \omega t)\right)
\end{aligned}
$$

Two lock-in amplifiers are connected to this output, one detecting at $\omega$ and the other at $2 \omega$. The detected intensities become

$$
\begin{aligned}
I_{D C} & =\frac{I_{0}}{4} a \\
I_{\omega} & =-\frac{I_{0}}{4}\left[b \sin \left(\delta_{0}(\lambda)\right)+c \cos \left(\delta_{0}(\lambda)\right)\right] 2 J_{1}(A) \\
I_{2 \omega} & =\frac{I_{0}}{4}\left[b \cos \left(\delta_{0}(\lambda)\right)-c \sin \left(\delta_{0}(\lambda)\right)\right] 2 J_{2}(A)
\end{aligned}
$$

These equations can be used to relate the measured signal to circular birefringence and dichroism. Faraday rotation is magnetic circular birefringence, and so a sample that displays Faraday rotation or magnetic circular dichroism can be described as having a magnetic-field dependent circular birefringence or circular dichroism. If the sample does not display any other anisotropy, its normalized Mueller matrix can be approximated as ${ }^{46,47}$

$$
M_{C B / C D \text { Sample }}=\left(\begin{array}{cccc}
1 & 0 & 0 & C D \\
0 & 1 & C B & 0 \\
0 & -C B & 1 & 0 \\
C D & 0 & 0 & 1
\end{array}\right)
$$

Using definition of $a, b$ and $c$ (equations (15) - (17)), equations (35) and (36) become

$$
\begin{aligned}
I_{\omega} & =-I_{D C}\left[C B \sin \left(\delta_{0}(\lambda)\right)+C D \cos \left(\delta_{0}(\lambda)\right)\right] 2 J_{1}(A) \\
I_{2 \omega} & =I_{D C}\left[C B \cos \left(\delta_{0}(\lambda)\right)-C D \sin \left(\delta_{0}(\lambda)\right)\right] 2 J_{2}(A)
\end{aligned}
$$

With knowledge of $\delta_{0}(\lambda)$ it would now be possible to determine the circular birefringence and dichroism of a sample. Two possible approaches exist to this problem. The first, described in section 3.2, employs an ideal PEM approximation. The alternative is to characterize $\delta_{0}(\lambda)$, which is described in section 3.3. 


\subsection{Measuring circular birefringence and dichroism with an ideal PEM}

Under the assumption of an ideal PEM with little or no static strain $\left(\delta_{0}(\lambda) \approx 0\right)$, equations $(38)$ and $(39)$ simplify to

$$
\begin{aligned}
I_{\omega} & =I_{D C} \cdot C D \cdot 2 J_{1}(A) \\
I_{2 \omega} & =I_{D C} \cdot C B \cdot 2 J_{2}(A) \\
C D & =\frac{I_{\omega}}{I_{D C} 2 J_{1}(A)}=0.9630 \frac{I_{\omega}}{I_{D C}} \\
C B & =\frac{I_{2 \omega}}{I_{D C} 2 J_{2}(A)}=1.1579 \frac{I_{2 \omega}}{I_{D C}}
\end{aligned}
$$

These are the well-known results that linearly correlate the size of the observed signals on both lock-in amplifiers to the circular dichroism and birefringence present in the samples. ${ }^{52}$ Under this approximation, it is possible to measure e.g. Faraday rotation by detecting at $2 \omega$ without needing to take into account any circular dichroism present in the sample.

\subsection{Measuring circular birefringence and dichroism with a real PEM}

The previous section used the simplification that $\delta_{0}(\lambda) \approx 0$. For a real PEM, this is not always a correct simplification, and in order to verify it, it is necessary to measure a sample that meets certain requirements. A sample is required with no natural or magnetic circular dichroism, and no linear birefringence or dichroism. Such a sample is easily found, e.g. toluene. For this sample, ${ }^{46} m_{03}=0$ and $m_{13}=0$, and so $a=1, b=C B$ and $c=C D=0$. We can then divide equation (38) by equation (39)

$$
\begin{aligned}
\frac{I_{\omega}}{I_{2 \omega}} & =\frac{-I_{D C}\left[C B \sin \left(\delta_{0}(\lambda)\right)+C D \cos \left(\delta_{0}(\lambda)\right)\right] 2 J_{1}(A)}{I_{D C}\left[C B \cos \left(\delta_{0}(\lambda)\right)-C D \sin \left(\delta_{0}(\lambda)\right)\right] 2 J_{2}(A)} \\
\frac{I_{\omega}}{I_{2 \omega}} & =\frac{-\sin \left(\delta_{0}(\lambda)\right) J_{1}(A)}{\cos \left(\delta_{0}(\lambda)\right) J_{2}(A)} \\
\tan \left(\delta_{0}(\lambda)\right) & =\frac{I_{\omega} J_{2}(A)}{I_{2 \omega} J_{1}(A)}
\end{aligned}
$$

And because $J_{1}(2.4048)=0.5192$ and $J_{2}(2.4048)=0.4318$

$$
\tan \left(\delta_{0}(\lambda)\right)=0.8316 \frac{I_{\omega}}{I_{2 \omega}}
$$

For each wavelength where $I_{2 \omega} \neq 0$, it is now possible to calculate $\delta_{0}(\lambda)$. In order to induce $I_{2 \omega} \neq 0$, a magnetic field is applied, causing Faraday rotation. We performed the measurements from $350 \mathrm{~nm}$ to $880 \mathrm{~nm}$ on toluene in a quartz cuvette with an optical path length of $1 \mathrm{~cm}$, in magnetic fields up to 1 tesla. For each wavelength, the value detected by the lock-in amplifiers $\left(I_{\omega}\right.$ and $\left.I_{2 \omega}\right)$ was recorded for a discrete set of magnetic fields. Using equation (47) it is possible to determine $\delta_{0}$.

$\delta_{0}$ of the employed PEM shows a typical decline towards longer wavelengths (Figure 2). ${ }^{53}$ At wavelengths longer than $550 \mathrm{~nm} \delta_{0}$ is smaller than the detection limit. At shorter wavelengths it becomes significantly larger than 0 , invalidating the ideal PEM $\delta_{0} \approx 0$ approximation frequently used. At $350 \mathrm{~nm}, \delta_{0}>0.04 \mathrm{rad}$, resulting in $\sin \left(\delta_{0}\right)>0.04$, causing a signal "leakage" of the circular birefringence into the circular dichroism of more than $4 \%$. The effect of this leakage on measurements can be demonstrated by filling in equations (38) and (39) with the correct value of $\delta_{0}$, and deriving the actual Faraday rotation and MCD. When a magnetic field is applied to toluene, there is large circular birefringence and no circular dichroism. Taking into account PEM non-idealities, the measured Faraday rotation is almost unchanged from the ideal PEM approximation $(<0.1 \%)$. The MCD is however greatly influenced, changing from an apparent nonzero signal, increasing with decreasing wavelength, to a zero signal (Figure 3). This false positive illustrates the importance of taking PEM non-idealities into account, particularly at shorter wavelengths. A small MCD contribution at a short wavelength can be entirely indiscernible due to the leakage of Faraday rotation signal into the MCD channel. 


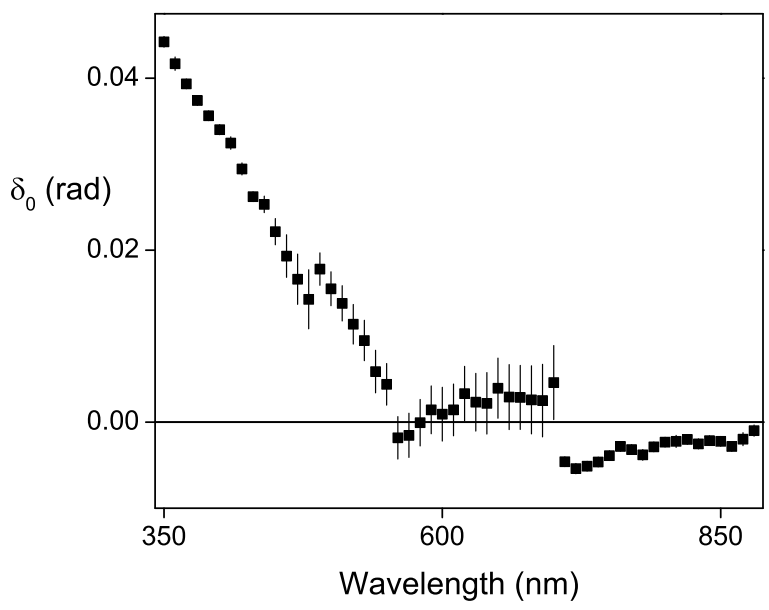

Figure 2. $\delta_{0}$ of the employed PEM shows a typical decline towards longer wavelengths. At wavelengths longer than $550 \mathrm{~nm}$ $\delta_{0}$ is smaller than the detection limit. At shorter wavelengths it becomes significantly larger than 0 , invalidating the ideal PEM approximation $\left(\delta_{0} \approx 0\right)$.

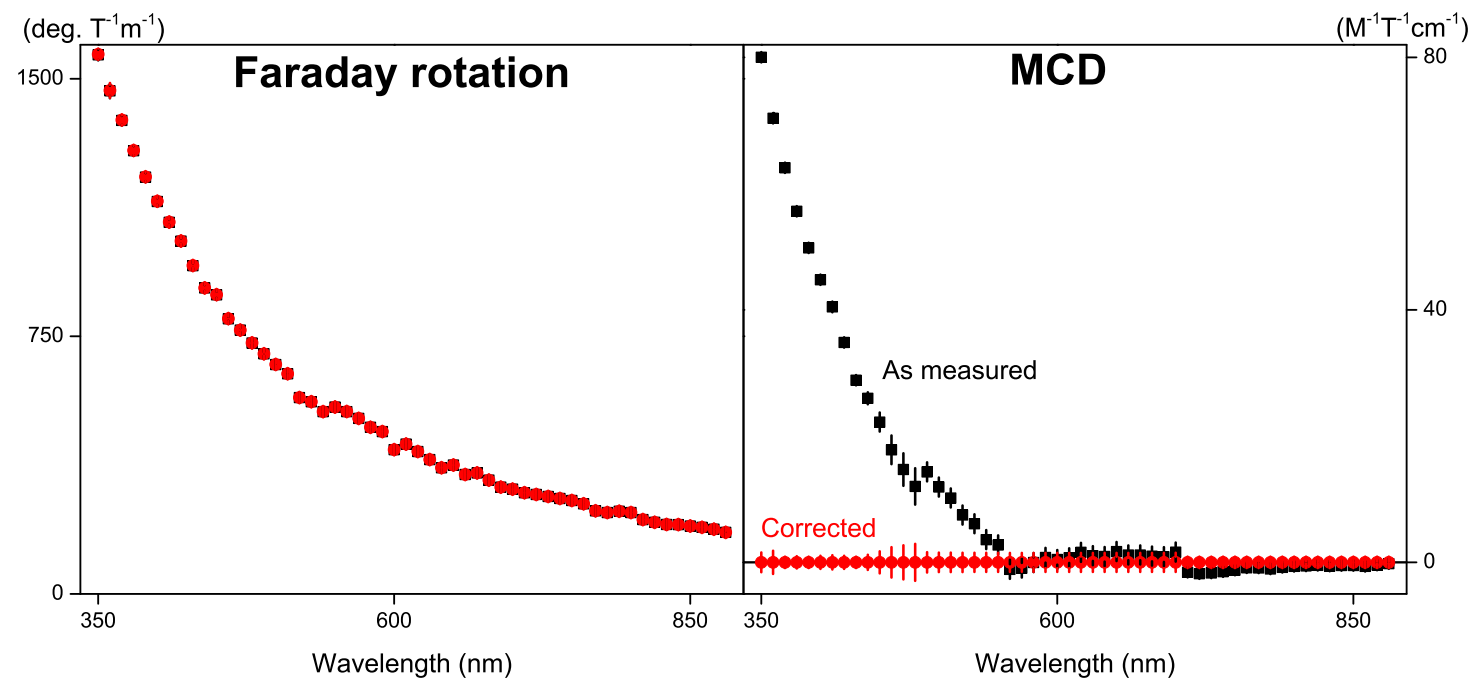

Figure 3. Correcting the measured signals for PEM non-idealities reveals significant differences in the data, which is even more clear when one of the signals is at least an order of magnitude larger than the other. 


\section{DISCUSSION AND PERSPECTIVES}

Controlling the polarization of light is an important domain of research. In order to be able to (magneto-) optically characterize novel materials to control the polarization of light, we designed a single PEM experimental setup. We theoretically analyzed and experimentally characterized the effect of non-idealities in the PEM on our data. The theoretical results can be summarized by equations (38) and (39), from which it is apparent that PEM non-idealities cause leakage of signal from the circular dichroism to the circular birefringence channel and vice versa. For our PEM, $\delta_{0}$, calculated via equations (38) and (39) from measurements on toluene, increases towards shorter wavelengths, reaching a value of more than $0.04 \mathrm{rad}$ at $350 \mathrm{~nm}$, invalidating the ideal PEM approximation.Because $\sin \left(\delta_{0}\right)>0.04$, a leakage of $\mathrm{CD}$ signal into the $\mathrm{CB}$ channel and vice versa occurs, at more than $4 \%$. At these values, the ideal PEM approximation to measure Faraday rotation or circular dichroism no longer holds, and the effect of $\delta_{0}$ on the signal must be taken into account.

Our conclusion also holds for more general setups such as CD spectrometers. Leakage between channels becomes more important towards shorter wavelengths, and can even dominate the measurements when CD and CB differ by more than an order of magnitude.

We have demonstrated the necessity to take PEM non-idealities into account when working with PEMs, especially at shorter wavelengths or when dealing with signals spanning different orders of magnitude. PEM non-idealities, while frequently neglected in experimental setup design and theoretical derivations, are expected to be more complicated and possibly exert a larger influence on obtained results for experimental setups with multiple PEMs.

\section{ACKNOWLEDGMENTS}

Stefaan Vandendriessche is grateful for the financial support from the FWO-Vlaanderen. We are grateful to the University of Leuven (GOA) for financial support. We acknowledge useful dicussions with Maarten Bloemen, Ward Brullot, Pieter-Jan Demeyer, Ventsislav K. Valev and Maarten Vanbel. 


\section{REFERENCES}

[1] Hecht, E., [Optics], Pearson Education (2008).

[2] Ginzburg, P., Fortuño, F. J. R., Wurtz, G. A., Dickson, W., Murphy, A., Morgan, F., Pollard, R. J., Iorsh, I., Atrashchenko, A., Belov, P. A., Kivshar, Y. S., Nevet, A., Ankonina, G., Orenstein, M., and Zayats, A. V., "Manipulating polarization of light with ultrathin epsilon-near-zero metamaterials," Opt. Express 21, 14907-14917 (2013).

[3] Zhao, Y. and Alù, A., "Manipulating light polarization with ultrathin plasmonic metasurfaces," Physical Review B 84, 205428- (2011).

[4] Kauranen, M., Van Elshocht, S., Verbiest, T., and Persoons, A., "Tensor analysis of the second-order nonlinear optical susceptibility of chiral anisotropic thin films," The Journal of Chemical Physics 112, 1497-1502 (2000).

[5] Kleinlogel, S. and White, A. G., "The secret world of shrimps: Polarisation vision at its best," PLoS ONE 3, e2190 EP - (2008).

[6] Vedel, M., Lechocinski, N., and Breugnot, S., "3d shape reconstruction of optical element using polarization," in [Polarization: Measurement, Analysis, and Remote Sensing IX, 767203], Chenault, D. B. and Goldstein, D. H., eds., 767203-767203, SPIE (2010).

[7] Vanbel, M. K., Valev, V. K., Vincent, B., Afanas'ev, V. V., Locquet, J.-P., Elshocht, S. V., Caymax, M., and Verbiest, T., "Second-harmonic generation reveals the oxidation steps in semiconductor processing," Journal of Applied Physics 111(6), 064504 (2012).

[8] Cohen, B., Martin, C., Iyer, S. K., Wiesner, U., and Douhal, A., "Single dye molecule behavior in fluorescent core-shell silica nanoparticles," Chemistry of Materials 24, 361-372 (2011).

[9] Jordan, D. S., Hull, C. J., Troiano, J. M., Riha, S. C., Martinson, A. B. F., Rosso, K. M., and Geiger, F. M., "Second harmonic generation studies of $\mathrm{Fe}(\mathrm{II})$ interactions with hematite $\left(\alpha-\mathrm{Fe}_{2} \mathrm{O}_{3}\right)$," The Journal of Physical Chemistry C 117, 4040-4047 (2013).

[10] lafelice, V. J. and Bickel, W. S., "Polarized light-scattering matrix elements for select perfect and perturbed optical surfaces," Appl. Opt. 26, 2410-2415 (1987).

[11] Guo, C.-S., Yue, S.-J., Wang, X.-L., Ding, J., and Wang, H.-T., "Polarization-selective diffractive optical elements with a twisted-nematic liquid-crystal display," Appl. Opt. 49, 1069-1074 (2010).

[12] Hasman, E., Kleiner, V., Biener, G., and Niv, A., "Polarization dependent focusing lens by use of quantized pancharatnam-berry phase diffractive optics," Applied Physics Letters 82, 328-330 (2003).

[13] Yang, D., Canit, J. C., and Gaignebet, E., "Photoelastic modulator: polarization modulation and phase modulation," Journal of Optics 26(4), 151 (1995).

[14] Kemp, J. C., "Piezo-optical birefringence modulators: New use for a long-known effect," J. Opt. Soc. Am. 59, 950-953 (1969).

[15] Hall, S. A., Covert, P. A., Blinn, B. R., Shakeri, S., and Hore, D. K., "Rapid and sensitive polarization measurement for characterizing protein adsorption at the solid-liquid interface," The Journal of Physical Chemistry C 117, 1796-1803 (2013).

[16] Nunez-Olvera, O., Balderas-Navarro, R. E., Ortega-Gallegos, J., Guevara-Macias, L. E., Armenta-Franco, A., Lastras-Montano, M. A., Lastras-Martinez, L. F., and Lastras-Martinez, A., "A rapid reflectancedifference spectrometer for real-time semiconductor growth monitoring with sub-second time resolution," Review of Scientific Instruments 83(10), 103109 (2012).

[17] Chandani, A., Fukuda, A., Kumar, S., and Vij, J. K., "Discovery of a novel ferrielectric phase of five-layer periodicity in binary mixtures of chiral smectic liquid crystals exhibiting unusual reversed phase sequence," Liquid Crystals 38(5), 663-668 (2011).

[18] Maisonneuve, M., Song, I.-H., Patskovsky, S., and Meunier, M., "Polarimetric total internal reflection biosensing," Opt. Express 19, 7410-7416 (2011).

[19] Han, C.-Y., Lai, C.-W., Chao, Y.-F., Leou, K.-C., and Lin, T.-L., "Assessment of interface roughness during plasma etching through the use of real-time ellipsometry," Applied Surface Science 257, 2536-2539 (2011).

[20] Kim, M.-H., Kurz, V., Acbas, G., Ellis, C. T., and Cerne, J., "Measurement of the infrared complex faraday angle in semiconductors and insulators," J. Opt. Soc. Am. B 28, 199-207 (2011). 
[21] Roedel, E., Urakawa, A., and Baiker, A., "In situ pm-irras study of powder catalyst: Dynamic evolutions of species on catalyst and in gas phase during nox storage-reduction," Catalysis Today 155, 172-176 (2010).

[22] Vandendriessche, S., Van Cleuvenbergen, S., Willot, P., Hennrich, G., Srebro, M., Valev, V. K., Koeckelberghs, G., Clays, K., Autschbach, J., and Verbiest, T., "Giant Faraday rotation in mesogenic organic molecules," Chemistry of Materials 25, 1139-1143 (2013).

[23] Cook, P. J., Zhang, J., Liu, Y., Guan, W., Wang, N., Qin, L., Shen, T. H., Jones, G. A., and Grundy, P. J., "Magneto-optical stokes polarimetry and nanostructured magnetic materials," Journal of Nanoscience and Nanotechnology 12(2), 1067-1073 (2012).

[24] Joshua, A. and Venkataraman, V., "Enhanced sensitivity in detection of kerr rotation by double modulation and time averaging based on allan variance," Review of Scientific Instruments 80(2), 023908 (2009).

[25] Xia, T. K., Hui, P. M., and Stroud, D., "Theory of faraday rotation in granular magnetic materials.," Journal of Applied Physics 67 (1990).

[26] Chin, J. Y., Steinle, T., Wehlus, T., Dregely, D., Weiss, T., Belotelov, V. I., Stritzker, B., and Giessen, H., "Nonreciprocal plasmonics enables giant enhancement of thin-film faraday rotation," Nat Commun 4, 1599 (2013).

[27] Suwa, M., Miyamoto, K., and Watarai, H., "Faraday rotation dispersion measurements of diamagnetic organic liquids and simultaneous determination of natural optical rotatory dispersion using a pulsed magnetic field," Analytical Sciences 29(1), 113-119 (2013).

[28] Vandendriessche, S., Valev, V. K., and Verbiest, T., "Faraday rotation and its dispersion in the visible region for saturated organic liquids," Phys. Chem. Chem. Phys. 14, 1860-1864 (2012).

[29] Faraday, M., [Experimental Researches in Electricity], vol. III, R. Taylor \& W. Francis (1839-1855).

[30] Bradley, J. M., Butt, J. N., and Cheesman, M. R., "Electrochemical titrations and reaction time courses monitored in situ by magnetic circular dichroism spectroscopy," Analytical Biochemistry 419, 110-116 (2011).

[31] Wagnière, G. H. and Rikken, G. L. J. A., "Chirality and magnetism: Free electron on an infinite helix, ncd, mcd, and magnetochiral dichroism," Chemical Physics Letters 481, 166-168 (2009).

[32] Kjaergaard, T., Kristensen, K., Kauczor, J., Jørgensen, P., Coriani, S., and Thorvaldsen, A. J., "Comparison of standard and damped response formulations of magnetic circular dichroism," The Journal of Chemical Physics 135, 024112-16 (2011).

[33] Botek, E., Champagne, B., Verbiest, T., Gangopadhyay, P., and Persoons, A., "A joint theoreticalexperimental investigation of the faraday effect in benzene, toluene, and p-xylene," ChemPhysChem $\mathbf{7}(8)$, 1654-1656 (2006).

[34] Bera, S. C. and Chakraborty, S., "Study of magneto-optic element as a displacement sensor," Measurement 44, 1747-1752 (2011).

[35] Nelissen, J., Nuyts, K., De Zotti, M., Lavigne, R., Lamberigts, C., and De Borggraeve, W. M., "Total synthesis of Septocylindrin B and C-terminus modified analogues," PLoS ONE 7, e51708 (2012).

[36] Farrag, M., Tschurl, M., and Heiz, U., "Chiral gold and silver nanoclusters: Preparation, size selection, and chiroptical properties," Chemistry of Materials 25, 862-870 (2013).

[37] Gu, Y. and Kornev, K. G., "Plasmon enhanced direct and inverse faraday effects in non-magnetic nanocomposites," J. Opt. Soc. Am. B 27, 2165-2173 (2010).

[38] Brullot, W., Reddy, N. K., Wouters, J., Valev, V. K., Goderis, B., Vermant, J., and Verbiest, T., "Versatile ferrofluids based on polyethylene glycol coated iron oxide nanoparticles," J. Magn. Magn. Mater. 324, 1919-1925 (2012).

[39] Bloemen, M., Brullot, W., Luong, T., Geukens, N., Gils, A., and Verbiest, T., "Improved functionalization of oleic acid-coated iron oxide nanoparticles for biomedical applications," Journal of Nanoparticle Research 14(9), 1-10 (2012).

[40] Barnakov, Y. A., Scott, B. L., Golub, V., Kelly, L., Reddy, V., and Stokes, K. L., "Spectral dependence of faraday rotation in magnetite-polymer nanocomposites," Journal of Physics and Chemistry of Solids $\mathbf{6 5}$, 1005-1010 (2004). 
[41] Jans, H., Jans, K., Demeyer, P.-J., Knez, K., Stakenborg, T., Maes, G., and Lagae, L., "A simple doublebead sandwich assay for protein detection in serum using uv-vis spectroscopy," Talanta 83, 1580-1585 (2011).

[42] Sempels, W., De Dier, R., Mizuno, H., Hofkens, J., and Vermant, J., "Auto-production of biosurfactants reverses the coffee ring effect in a bacterial system," Nat Commun 4, 1757 (2013).

[43] Bloemen, M., Brullot, W., Denis, C., Vanysacker, L. and Verbiest, T. "Core-shell nanoparticles as enhanced probes for imaging applications," in [Biophotonics: Photonic Solutions for Better Health Care III], Popp, J., Drexler, W., Tuchin, V. V. and Matthews, D. L., eds., 84272Q, SPIE, SPIE (2012).

[44] Demeyer, P. J., Bloemen, M., Verbiest, T., and Clays, K., "Tuning the properties of colloidal magnetophotonic crystals by controlled infiltration with superparamagnetic magnetite nanoparticles," in [Photonic Crystal Materials and Devices X, 84251R], Míguez, H. R., Romanov, S. G., Andreani, L. C., and Seassal, C., eds., 84251R, SPIE (2012).

[45] Castiglioni, E., Albertini, P., and Abbate, S., "Evaluation of instrumental errors built in circular dichroism spectrometers," Chirality 22(1E), E142-E148 (2010).

[46] Takechi, H., Arteaga, O., Ribo, J. M., and Watarai, H., "Chiroptical measurement of chiral aggregates at liquid-liquid interface in centrifugal liquid membrane cell by mueller matrix and conventional circular dichroism methods," Molecules 16(5), 3636-3647 (2011).

[47] Arteaga, O. and Canillas, A., "Pseudopolar decomposition of the jones and mueller-jones exponential polarization matrices," J. Opt. Soc. Am. A 26, 783-793 (2009).

[48] Hu, C. G., Sun, L. D., Li, Y. N., Hohage, M., Flores-Camacho, J. M., Hu, X. T., and Zeppenfeld, P., "Retardation correction for photoelastic modulator-based multichannel reflectance difference spectroscopy," J. Opt. Soc. Am. A 25, 1240-1245 (2008).

[49] Ghosh, N., Wood, M. F. G., and Vitkin, I. A., "Mueller matrix decomposition for extraction of individual polarization parameters from complex turbid media exhibiting multiple scattering, optical activity, and linear birefringence," Journal of Biomedical Optics 13(4), 044036 (2008).

[50] Bickel, W. S. and Bailey, W. M., "Stokes vectors, mueller matrices, and polarized scattered light," American Journal of Physics 53, 468-478 (1985).

[51] Tompkins, H. and Irene, E., [Handbook of ellipsometry], William Andrew Pub. (2005).

[52] Wallace, B. and Janes, R., [Modern techniques for circular dichroism and synchrotron radiation circular dichroism spectroscopy], IOS Press (2009).

[53] Jellison, G. E. and Modine, F. A., "Two-modulator generalized ellipsometry: experiment and calibration," Appl. Opt. 36, 8184-8189 (1997). 